\title{
Causality in Statistical Power: Isomorphic Properties of Measurement, Research Design, Effect Size, and Sample Size
}

\author{
R. Eric Heidel \\ Department of Surgery, Office of Medical Education, Research, and Development, University of Tennessee Graduate School of Medicine, \\ 1924 Alcoa Highway, Knoxville, TN 37920, USA
}

Correspondence should be addressed to R. Eric Heidel; rheidel@utmck.edu

Received 29 December 2015; Accepted 3 March 2016

Academic Editor: G. Bryan Young

Copyright (c) 2016 R. Eric Heidel. This is an open access article distributed under the Creative Commons Attribution License, which permits unrestricted use, distribution, and reproduction in any medium, provided the original work is properly cited.

\begin{abstract}
Statistical power is the ability to detect a significant effect, given that the effect actually exists in a population. Like most statistical concepts, statistical power tends to induce cognitive dissonance in hepatology researchers. However, planning for statistical power by an a priori sample size calculation is of paramount importance when designing a research study. There are five specific empirical components that make up an a priori sample size calculation: the scale of measurement of the outcome, the research design, the magnitude of the effect size, the variance of the effect size, and the sample size. A framework grounded in the phenomenon of isomorphism, or interdependencies amongst different constructs with similar forms, will be presented to understand the isomorphic effects of decisions made on each of the five aforementioned components of statistical power.
\end{abstract}

\section{Introduction}

Statistical power, like statistics in general, tends to induce feelings of cognitive dissonance in people [1]. Statistical power is the ability to detect treatment effects, given that they truly exist in the population [2]. In more general terms, statistical power is the chance that researchers will achieve a significant $p$ value. In the applied sense, hepatology researchers must balance several different empirical factors that have causal and isomorphic effects on statistical power.

Isomorphism is the phenomenon where constructs that are different in content, but similar in form, are linked due to their interdependent associations [3]. The constructs of measurement, research design, magnitude and variance of effect size, and sample size are all isomorphic in their effects on statistical power and each other. A change in one construct will cause a predictable change in statistical power, as well as the other respective constructs. The etymology of the word isomorphism comes from the Greek "isos," meaning equal, and "morphe," meaning form [4]. In essence, the constructs of measurement, research design, magnitude and variance of effect size, and sample size are equal in their causal effects on the formation of statistical power in applied research.
A biostatistician at a regional medical center in southeastern United States conducted several thousand consultations with residents, fellows, faculty, physicians, and staff over the course of eight years. A priori sample size calculations continually proved to be the hardest part of assisting novice and expert researchers in the planning stages of conducting research. Consultees oftentimes had no idea what type of effect size should be detected in their respective studies. The biostatistician created a conceptual framework grounded in isomorphism, or the interdependencies that exist amongst different constructs, to better understand how the choices made by consultees had causal effects on statistical power. These isomorphic relationships are presented in Table 1.

\section{Methods}

In order to conduct an a priori sample size calculation to achieve adequate statistical power, hepatology researchers must make decisions about (1) the scale of measurement of the outcome, (2) the research design, (3) the magnitude of the effect size, (4) the variance of the effect size, and (5) the sample size that can feasibly be collected [2]. Researchers 
TABLE 1: Isomorphism and causal effects on statistical power.

\begin{tabular}{lcc}
\hline Empirical component & Choice & Effect on statistical power \\
\hline Measurement of outcome & Categorical outcome & Decreased statistical power \\
& Ordinal outcome & $\begin{array}{c}\text { Decreased statistical power } \\
\text { Increased statistical power }\end{array}$ \\
\hline \multirow{2}{*}{ Research design } & Continuous outcome & Decreased statistical power \\
& Within-subjects & Increased statistical power \\
Magnitude of effect size & Multivariate & Decreased statistical power \\
\hline \multirow{2}{*}{ Variance of effect size } & Small effect size & Decreased statistical power \\
& Large effect size & Increased statistical power \\
\hline \multirow{2}{*}{ Sample size } & Homogeneity & Increased statistical power \\
& Heterogeneity & Decreased statistical power \\
\hline
\end{tabular}

further have to understand how their choices will ultimately affect statistical power. Decisions made by researchers related to these five constructs will have causal effects on statistical power. The underlying isomorphic reasoning associated with making decisions related to the five empirical components when conducting an a priori sample size calculation was presented. The strengths and weaknesses of decisions made by researchers in regard to each of the five components were identified.

\section{Results}

3.1. Statistical Power and Measurement of Outcomes. Measurement plays a central role in the ability to detect significant treatment effects [2]. Precision and accuracy in measurement leads to more statistical power. Continuous level measurement of outcomes (interval, ratio, and count) leads to more statistical power and smaller sample sizes. More powerful parametric statistics are used with continuous outcomes. There is inherent measurement error and lack of precision in ordinal and categorical outcomes, leading to decreased statistical power and larger sample sizes required to detect significant effects. Less powerful nonparametric statistics are used with these types of outcomes.

3.2. Statistical Power and Research Designs. Research designs are used to answer research questions [5]. However, withinsubjects designs provide much more statistical power and significantly smaller sample sizes in applied research. The increase in statistical power comes from participants serving as their own controls. Between-subjects designs decrease statistical power and require larger sample sizes to detect significant effects between independent groups. Multivariate designs will always decrease statistical power and necessitate larger sample sizes to be able to detect confounding effects [6].

3.3. Statistical Power and Magnitude of Effect Size. Planning for the effect size is perhaps the hardest part of planning a research study [2]. First, researchers have to specify the scale of measurement of the outcome to know if they are detecting differences in proportions or means and standard deviations. Then, depending upon the research design being used, researchers hypothesize their effect sizes between independent groups, within subjects, or in a multivariate fashion.

The best choice, by far, when planning an a priori sample size calculation is to use the means, standard deviations, proportions, and odds ratios presented in the empirical literature. This is called using an evidence-based measure of effect size in applied research. Researchers can increase the internal validity of their studies by using effect sizes reported in the literature [5]. Using an evidence-based measure of effect size also demonstrates more empirical rigor on their part to plan for a valid effect size and achieve adequate statistical power.

There are two components of an effect size that have isomorphic relationships with statistical power: the magnitude of the effect size and the variance of the effect size [5]. The magnitude of an effect size is the relative difference or change in an outcome expected as a result of treatment or group membership. The variance of an effect size is defined as the amount of homogeneity (or heterogeneity) that is expected in an outcome as a result of treatment.

Large effect sizes will increase statistical power and decrease the needed sample size. Larger effect sizes are easier to detect and require fewer observations if the hypothesized effect holds true in the population. Small effect sizes will always decrease statistical power and increase the needed sample size. In order to detect the nuances of variance within a small effect size, significantly more observations of the outcome will be needed to obtain adequate statistical power.

3.4. Statistical Power and Variance of Effect Size. Homogeneity or limited variance in an outcome will increase statistical power and decrease the needed sample size. Group differences or changes in outcomes will be more easily detected in groups of participants that are similar. Inversely, 
heterogeneity or extensive variance in an outcome reduces the ability to detect significant effects due to such wide dispersion of outcomes in groups or across time.

In applied statistical practice, researchers should overestimate the variance of a given effect size. Overestimating the variance of an effect forces researchers to collect larger sample sizes. While this is not always feasible, the benefits of the overestimation are twofold. Statistical power increases as the sample size grows larger and the precision and accuracy of the treatment effect is greatly improved. In theory, more of the diversity and variance in a given population is represented with larger sample sizes, leading to more generalizable statistical findings [7].

3.5. Statistical Power and Sample Size. With larger sample sizes, the chances of detecting significant effects increase drastically. Larger sample sizes allow researchers to detect both small and large effect sizes, regardless of their respective variances. Large sample sizes allow for flexibility in detecting treatment effects. Large sample sizes increase flexibility by being able to detect a wide spectrum of effect sizes (small, large, homogeneous, and heterogeneous). Small sample sizes significantly decrease statistical power and decrease the flexibility of detecting any type of effect size. With larger sample sizes, validation techniques such as bootstrapping, split-group, and jack-knife methods can be used to increase precision and accuracy of statistical findings [8].

\section{Discussion}

Statistical power is critical when conducting hepatology research. In order to bolster the understanding of this nebulous and cognitive dissonance-inducing construct in hepatology research, isomorphism can be applied as a framework to make better decisions when designing observational and experimental research. Causality in statistical power is associated with the interdependent and isomorphic relationships between measurement, research design, effect size, and sample size. The decisions made by researchers in regard to the aforementioned constructs will have causal effects on statistical power.

The decisions made by hepatology researchers are completely dependent upon the research question being asked and the current empirical environment. Sometimes, researchers will have to make decisions that they know will decrease statistical power. Many outcomes of interest in medicine are measured at a categorical level. Betweensubjects designs are important for understanding group differences related to treatment effects. Multivariate designs can account for confounding variables when predicting outcomes. Small effect sizes that exist in heterogeneous populations may push a clinician past a test or treatment threshold. Small sample sizes may be the only feasible choice when researching rare types of outcomes and disease states. However, the most important thing for researchers to remember is that statistical power is influenced in a causal manner by the decisions made when conducting an a priori sample size calculation. Isomorphism is a framework that can assist researchers in designing the most powerful studies given the current research question and research environment.

4.1. Scoring Methodology for Statistical Power. While writing the paper, the authors designed a scoring rubric containing every combination of scale of measurement, research design, magnitude, variance, and sample size. This rubric can be used to identify combinations of these five isomorphic constructs that lead to feasible and powerful research designs. The scoring methodology has not been validated formally in the literature but presents a pretty straightforward and userfriendly method for "grading" the combinations of decisions made by researchers in the preliminary phases of a study. A total of 72 possible combinations of the five empirical constructs were identified.

In terms of scoring in the instrument, if the chosen scale of measurement of the outcome was categorical or ordinal, a "0" was given. If the outcome was measured at a continuous level, then a " 1 " is given. This is because increased precision and accuracy in continuous level measurement increases statistical power. For research designs, a value of " 0 " is given to between-subjects designs and multivariate designs and "1" is given to the within-subjects design because each participant serves as their own control, leading to more statistical power. With magnitude of effect size, " 0 " is assigned to a small effect size and " 1 " denotes a large effect size, because large effect sizes are easier to detect, thus increasing power. Limited variance of outcome is coded as "1" because homogeneity leads to clear delineations between independent groups. Heterogeneity is coded as "0" because it is harder to detect effects in highly diverse populations, decreasing statistical power. Finally, a large sample size was coded as " 1 " because large sample sizes increase statistical power and increase the ability to detect all kinds of effect sizes. Small sample sizes were coded as "0." All the 72 rows were summed and statistical power values were created. The score ranges from 0 to 5 with increasing values denoting more statistical power as per the decisions made by researchers. The rating scale for 72 different research designs with respective statistical power scores is presented in Table 2.

\section{Conclusion}

The choices that hepatology researchers make related to measurement of outcomes, research design, magnitude and variance of effect size, and sample size have causal effects on statistical power in applied research. Isomorphism can be used as a framework to increase the understanding of the effects of decisions made by researchers on statistical power. Researchers will be able to make more informed decisions related to a priori sample size calculations and design more powerful studies.

\section{Competing Interests}

The author declares that there are no competing interests. 
TABLE 2: Scoring methodology for statistical power.

\begin{tabular}{|c|c|c|c|c|c|c|}
\hline Study & Outcome & Research design & Magnitude & Variance & Sample size & Score \\
\hline 1 & Categorical & Between-subjects & Small & Heterogeneity & Small & 0 \\
\hline 2 & Categorical & Multivariate & Small & Heterogeneity & Small & 0 \\
\hline 3 & Ordinal & Between-subjects & Small & Heterogeneity & Small & 0 \\
\hline 4 & Ordinal & Multivariate & Small & Heterogeneity & Small & 0 \\
\hline 5 & Categorical & Within-subjects & Small & Heterogeneity & Small & 1 \\
\hline 6 & Categorical & Between-subjects & Small & Heterogeneity & Large & 1 \\
\hline 7 & Categorical & Multivariate & Small & Heterogeneity & Large & 1 \\
\hline 8 & Categorical & Between-subjects & Small & Homogeneity & Small & 1 \\
\hline 9 & Categorical & Multivariate & Small & Homogeneity & Small & 1 \\
\hline 10 & Categorical & Between-subjects & Small & Homogeneity & Large & 1 \\
\hline 11 & Categorical & Multivariate & Small & Homogeneity & Large & 1 \\
\hline 12 & Categorical & Between-subjects & Large & Heterogeneity & Small & 1 \\
\hline 13 & Categorical & Multivariate & Large & Heterogeneity & Small & 1 \\
\hline 14 & Ordinal & Within-subjects & Small & Heterogeneity & Small & 1 \\
\hline 15 & Ordinal & Between-subjects & Small & Heterogeneity & Large & 1 \\
\hline 16 & Ordinal & Multivariate & Small & Heterogeneity & Large & 1 \\
\hline 17 & Ordinal & Between-subjects & Small & Homogeneity & Small & 1 \\
\hline 18 & Ordinal & Multivariate & Small & Homogeneity & Small & 1 \\
\hline 19 & Ordinal & Between-subjects & Small & Homogeneity & Large & 1 \\
\hline 20 & Ordinal & Multivariate & Small & Homogeneity & Large & 1 \\
\hline 21 & Ordinal & Between-subjects & Large & Heterogeneity & Small & 1 \\
\hline 22 & Ordinal & Multivariate & Large & Heterogeneity & Small & 1 \\
\hline 23 & Continuous & Between-subjects & Small & Heterogeneity & Small & 1 \\
\hline 24 & Continuous & Multivariate & Small & Heterogeneity & Small & 1 \\
\hline 25 & Categorical & Within-subjects & Small & Heterogeneity & Large & 2 \\
\hline 26 & Categorical & Within-subjects & Small & Homogeneity & Small & 2 \\
\hline 27 & Categorical & Within-subjects & Small & Homogeneity & Large & 2 \\
\hline 28 & Categorical & Within-subjects & Large & Heterogeneity & Small & 2 \\
\hline 29 & Categorical & Between-subjects & Large & Heterogeneity & Large & 2 \\
\hline 30 & Categorical & Multivariate & Large & Heterogeneity & Large & 2 \\
\hline 31 & Categorical & Between-subjects & Large & Homogeneity & Small & 2 \\
\hline 32 & Categorical & Multivariate & Large & Homogeneity & Small & 2 \\
\hline 33 & Ordinal & Within-subjects & Small & Heterogeneity & Large & 2 \\
\hline 34 & Ordinal & Within-subjects & Small & Homogeneity & Small & 2 \\
\hline 35 & Ordinal & Within-subjects & Small & Homogeneity & Large & 2 \\
\hline 36 & Ordinal & Within-subjects & Large & Heterogeneity & Small & 2 \\
\hline 37 & Ordinal & Between-subjects & Large & Heterogeneity & Large & 2 \\
\hline 38 & Ordinal & Multivariate & Large & Heterogeneity & Large & 2 \\
\hline 39 & Ordinal & Between-subjects & Large & Homogeneity & Small & 2 \\
\hline 40 & Ordinal & Multivariate & Large & Homogeneity & Small & 2 \\
\hline 41 & Continuous & Within-subjects & Small & Heterogeneity & Small & 2 \\
\hline 42 & Continuous & Between-subjects & Small & Heterogeneity & Large & 2 \\
\hline 43 & Continuous & Multivariate & Small & Heterogeneity & Large & 2 \\
\hline 44 & Continuous & Between-subjects & Small & Homogeneity & Small & 2 \\
\hline 45 & Continuous & Multivariate & Small & Homogeneity & Small & 2 \\
\hline 46 & Continuous & Between-subjects & Small & Homogeneity & Large & 2 \\
\hline 47 & Continuous & Multivariate & Small & Homogeneity & Large & 2 \\
\hline 48 & Continuous & Between-subjects & Large & Heterogeneity & Small & 2 \\
\hline
\end{tabular}


TABLE 2: Continued.

\begin{tabular}{|c|c|c|c|c|c|c|}
\hline Study & Outcome & Research design & Magnitude & Variance & Sample size & Score \\
\hline 49 & Continuous & Multivariate & Large & Heterogeneity & Small & 2 \\
\hline 50 & Categorical & Within-subjects & Large & Heterogeneity & Large & 3 \\
\hline 51 & Categorical & Within-subjects & Large & Homogeneity & Small & 3 \\
\hline 52 & Categorical & Between-subjects & Large & Homogeneity & Large & 3 \\
\hline 53 & Categorical & Multivariate & Large & Homogeneity & Large & 3 \\
\hline 54 & Ordinal & Within-subjects & Large & Heterogeneity & Large & 3 \\
\hline 55 & Ordinal & Within-subjects & Large & Homogeneity & Small & 3 \\
\hline 56 & Ordinal & Between-subjects & Large & Homogeneity & Large & 3 \\
\hline 57 & Ordinal & Multivariate & Large & Homogeneity & Large & 3 \\
\hline 58 & Continuous & Within-subjects & Small & Heterogeneity & Large & 3 \\
\hline 59 & Continuous & Within-subjects & Small & Homogeneity & Small & 3 \\
\hline 60 & Continuous & Within-subjects & Small & Homogeneity & Large & 3 \\
\hline 61 & Continuous & Within-subjects & Large & Heterogeneity & Small & 3 \\
\hline 62 & Continuous & Between-subjects & Large & Heterogeneity & Large & 3 \\
\hline 63 & Continuous & Multivariate & Large & Heterogeneity & Large & 3 \\
\hline 64 & Continuous & Between-subjects & Large & Homogeneity & Small & 3 \\
\hline 65 & Continuous & Multivariate & Large & Homogeneity & Small & 3 \\
\hline 66 & Categorical & Within-subjects & Large & Homogeneity & Large & 4 \\
\hline 67 & Ordinal & Within-subjects & Large & Homogeneity & Large & 4 \\
\hline 68 & Continuous & Within-subjects & Large & Heterogeneity & Large & 4 \\
\hline 69 & Continuous & Within-subjects & Large & Homogeneity & Small & 4 \\
\hline 70 & Continuous & Between-subjects & Large & Homogeneity & Large & 4 \\
\hline 71 & Continuous & Multivariate & Large & Homogeneity & Large & 4 \\
\hline 72 & Continuous & Within-subjects & Large & Homogeneity & Large & 5 \\
\hline
\end{tabular}

Note. Higher scores denote more statistical power.

\section{Acknowledgments}

Many thanks are due to Mitchell Goldman, MDFACS, William Metheny, Ph.D., and James Neutens, Ph.D., at the University of Tennessee Graduate School of Medicine for their continued support.

\section{References}

[1] D. A. Zahn and D. J. Isenberg, "Nonstatistical aspects of statistical consulting," The American Statistician, vol. 37, no. 4, pp. 297-302, 1983.

[2] S. B. Hulley, S. R. Cummings, W. S. Browner, D. G. Grady, and T. B. Newman, Designing Clinical Research, Lippincott, Williams, \& Wilkins, Philadelphia, Pa, USA, 4th edition, 2013.

[3] D. R. Hofstadter, Gödel, Escher, Bach: An Eternal Golden Braid, Basic Books, New York, NY, USA, 1979.

[4] Isomorphism, "Merriam-Webster Online Dictionary," December 2015, http://www.merriam-webster.com/dictionary/isomorphism.

[5] M. H. Katz, Study Design and Statistical Analysis: A Practical Guide for Clinicians, Cambridge University Press, New York, NY, USA, 2013.

[6] M. H. Katz, Multivariable Analysis: A Practical Guide for Clinicians and Public Health Researchers, Cambridge University Press, New York, NY, USA, 3rd edition, 2011.
[7] M. H. Katz, Evaluating Clinical and Public Health Interventions: A Practical Guide to Study Design and Statistics, Cambridge University Press, New York, NY, USA, 2010.

[8] B. Dawson and R. G. Trapp, Basic and Clinical Biostatistics, McGraw-Hill, New York, NY, USA, 4th edition, 2004. 


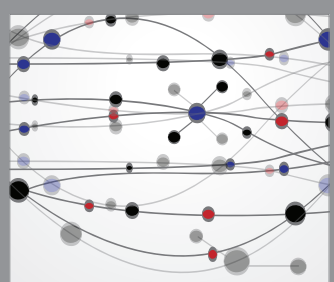

The Scientific World Journal
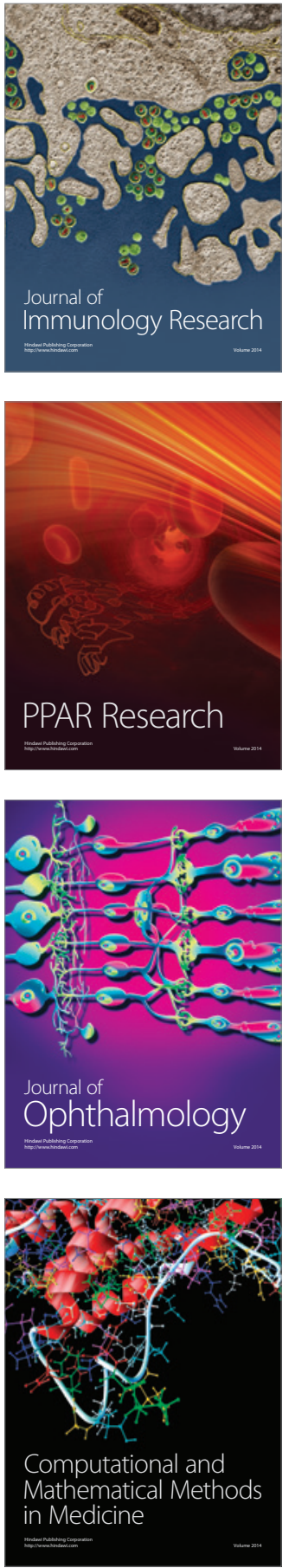

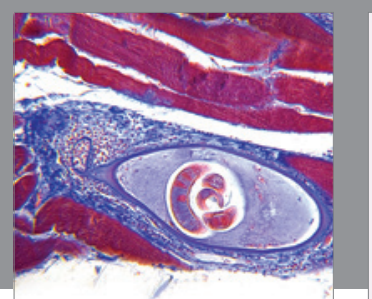

Gastroenterology Research and Practice

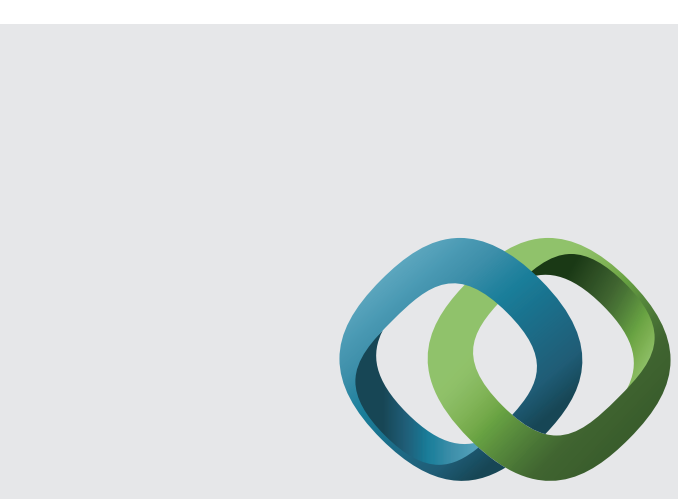

\section{Hindawi}

Submit your manuscripts at

http://www.hindawi.com
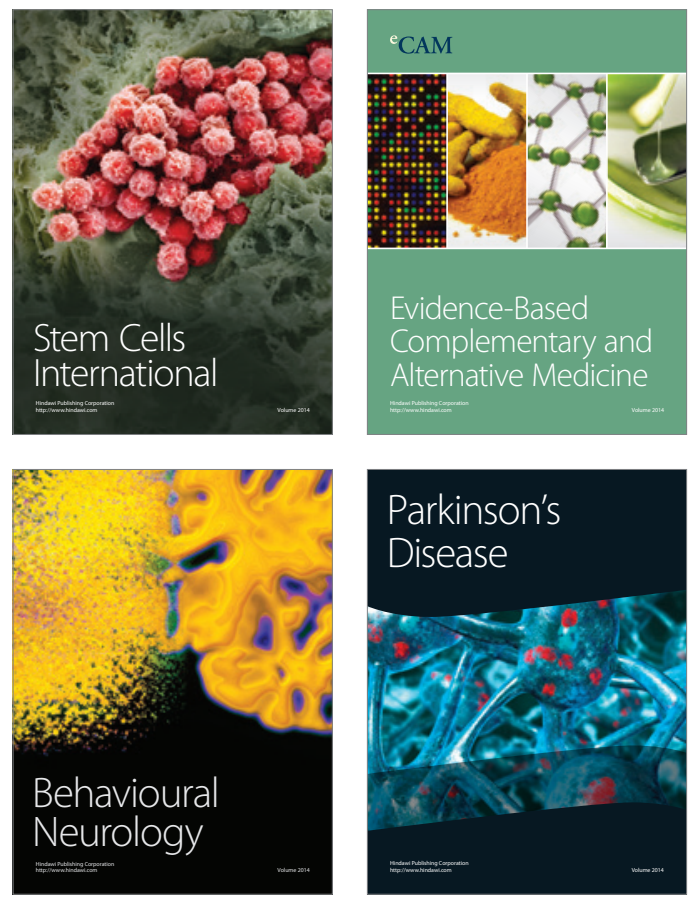
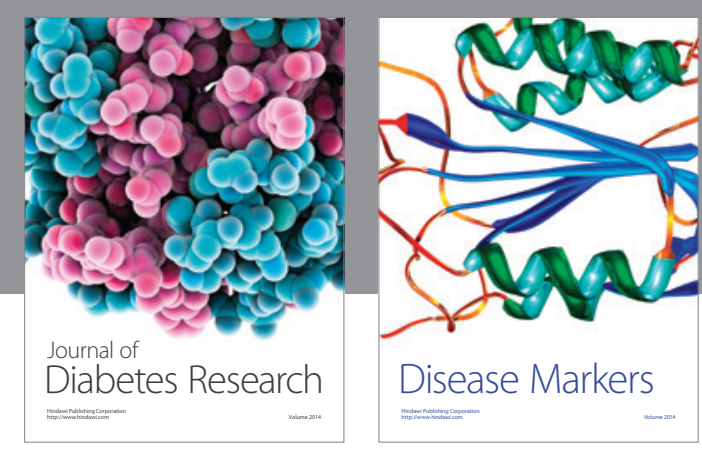

Disease Markers
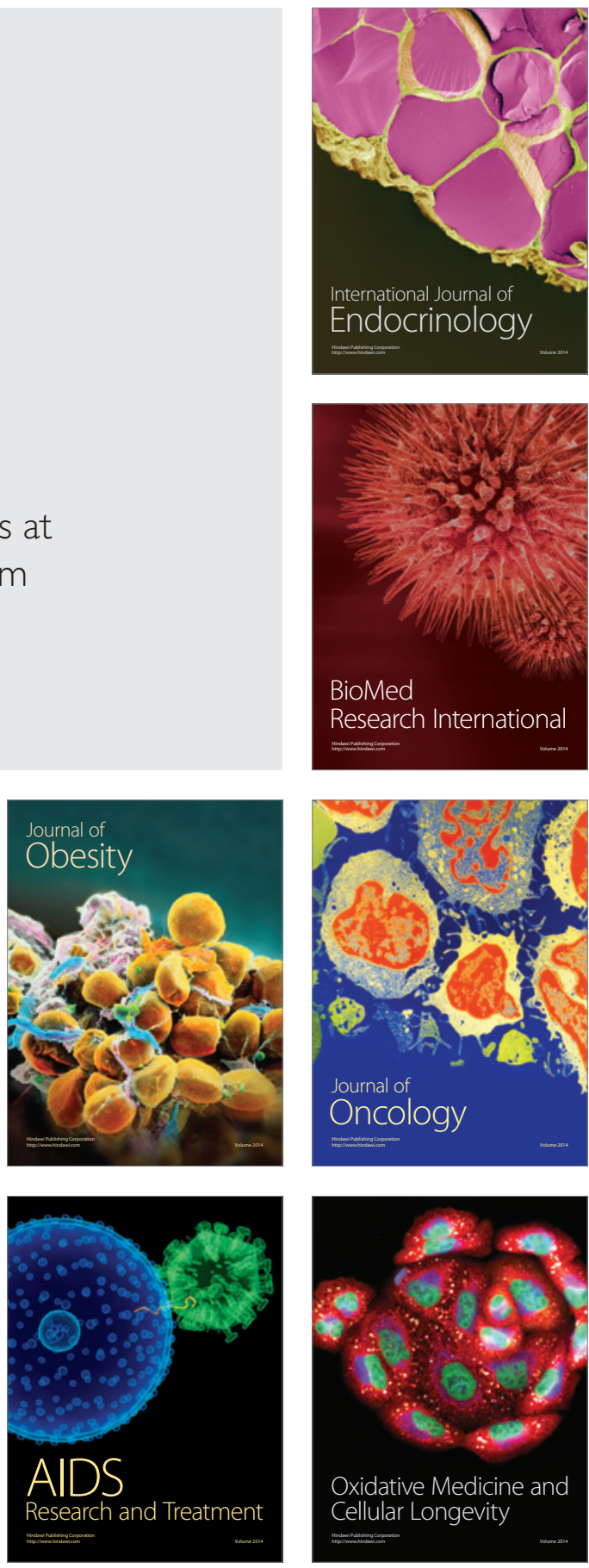